\title{
Potencial da Técnica in Vitro Semi-Automática de Produção de Gases para Avaliação de Silagens de Sorgo (Sorghum bicolor (L.) Moench)
}

\section{Rogério Martins Maurício, Luiz Gustavo Ribeiro Pereira², Lúcio Carlos Gonçalves ${ }^{3}$, Norberto Mário Rodriguez ${ }^{3}$, René Galvão Rezende Martins ${ }^{4}$, José Avelino Santos Rodrigues ${ }^{5}$}

RESUMO - O potential da técnica in vitro semi-automática de produção de gases foi estudado pela avaliação das silagens de quatro híbridos de sorgo (BR700, BR701, BR601 e AG2002). Os resultados desse experimento foram comparados aos obtidos em experimento de digestibilidade aparente. A relação entre a digestibilidade da matéria seca obtida pela técnica de produção de gases após 96 horas de fermentação (DMS) e a digestibilidade aparente da MS foi representada pela equação: digestibilidade in vivo $(\mathrm{g} / \mathrm{kg})=0,46 \mathrm{x} \mathrm{DMS}(\mathrm{g} / \mathrm{kg})$ $+361,08\left(\mathrm{r}^{2}=0,97\right)$. A técnica in vitro semi automática de produção de gases estimou de forma precisa os valores de digestibilidade aparente da MS das silagens avaliadas nesse experimento. Além disto, forneceu informações adicionais sobre a cinética de fermentação ruminal das silagens e degradabilidade efetiva da matéria seca em diferentes taxas de passagem. A superioridade da taxa de produção de gases $(\% / h)$ do híbrido BR601 (0,056) em relação ao BR700 (0,051), BR701 (0,044) e AG2002 (0,045) está correlacionada com a maior DMS do material $(649,598,601$ e $593 \mathrm{~g} / \mathrm{kg}$, respectivamente). Dessa forma, a técnica in vitro semi-automática de produção de gases foi capaz de selecionar o híbrido BR601, em termos de digestibilidade e cinética de fermentação ruminal, como o mais promissor para uso na alimentação dos ruminantes, demonstrando assim o seu potencial para avaliação de silagens de sorgo.

Palavras-chave: in vitro, produção de gases, ruminantes, valor nutritivo

\section{Potencial of Semi-Automated in Vitro Gas Production Technique for Sorghum Silages Evaluation}

\begin{abstract}
The potential of the semi-automated in vitro gas production technique was evaluated by incubation of four hybrids of sorghum (BR700, BR701, BR601 and AG2002). The results of this experiment (in vitro dry matter digestibility, DMD) were compared with the apparent in vivo digestibility. The relationship between the dry matter digestibility obtained by the gas production technique after 96 hours of incubation and the apparent dry matter digestibility in vivo was demonstrated by the equation: in vivo digestibility $(\mathrm{g} / \mathrm{kg})=0.46 \mathrm{x}$ DMD $(\mathrm{g} / \mathrm{kg})+361.08\left(\mathrm{r}^{2}=0.97\right)$. The gas technique estimated the apparent in vivo dry matter digestibility for all samples evaluated in this experiment. In addition, the rumen fermentation kinetics and also the dry matter extent of degradation using different rates of passage were calculated. The greater rate of gas production $(\% / \mathrm{h})$ obtained from the genotype BR601 (0.056) in relation to BR700 (0.051), BR701 (0.044) and AG2002 (0.045) was probably related to the higher DMD of the hybrid $(649,598,601 \mathrm{and} 593 \mathrm{~g} / \mathrm{kg}$ respectively). Therefore, the semi-automated in vitro gas production technique was able to select the best hybrid BR601 in terms of digestibility and kinetics of fermentation and consequently with higher nutritional value for ruminant nutrition.
\end{abstract}

Key Words: in vitro, gas production, nutritional value, ruminants

\section{Introdução}

A planta de sorgo, devido a facilidade de cultivo, altos rendimentos e produção de silagem de boa qualidade, é uma das espécies mais adaptadas à produção de silagem (Zago, 1999). Apresenta ainda uma importante característica de manter vivo o seu sistema radicular após a colheita para ensilagem, o que possibilita, em condições adequadas de temperatura, umidade e presença de nutrientes, uma rebrota com considerável produção de matéria seca (Casela et al., 1986; Zago, 1991).

A silagem de sorgo constitui um bom alimento para ser fornecido aos ruminates, apresentando valor nutricional bem próximo ao do milho, mas com produtividade de matéria seca por hectare superior.

\footnotetext{
${ }^{1}$ Pesquisador da FUNED (Fundação Ezequiel Dias), Rua Conde Pereira Carneiro 80, CEP: 30350-110, Belo Horizonte, MG. E.mail: rmmfuned@funed.mg.gov.br

2 Veterinário doutorando em Ciência Animal, Escola de Veterinária - UFMG, Av Antônio Carlos 6627, CEP: 30161-970, Caixa Postal 567,

Belo Horizonte, MG. E.mail: pereiralgr@hotmail.com

${ }^{3}$ Professores da Escola de Veterinária - UFMG, Av. Antônio Carlos 6627, CEP: 30161-970, Caixa Postal 567, Belo Horizonte, MG. E.mail: luciocg@vet.ufmg.br; norberto@vet.ufmg.br

${ }^{4}$ Estudante de doutorado - Departamento de Zootecnia da UFV, Viçosa-MG, CEP: 36571-000.

5 Pesquisador da EMBRAPA Milho e Sorgo. E.mail: avelino@cnpms.embrapa.br
} 
Além disso, é uma planta fisiologicamente bem adaptada às condições brasileiras, sendo uma boa opção para cultivo na época da safrinha.

Novos híbridos vêm sendo desenvolvidos e precisam ser avaliados por experimentos que incluam resposta animal (ex. digestibilidade, consumo e desempenho). Ensaios in vivo envolvendo produção animal e digestibilidade são os métodos mais adequados para determinar o valor nutricional dos alimentos utilizado na nutrição dos ruminantes. Entretanto, os mesmos requerem considerável uso de animais, alimentos, mão-deobra, tempo e alto custo financeiro, limitando assim a sua aplicabilidade. Como conseqüência, várias técnicas in vitro vêm sendo utilizadas como opção, devido ao baixo custo e rápida execução. Porém, as técnicas in vitro, para que tenham credibilidade, precisam ser validadas por meio de experimentos realizados in vivo.

A técnica de produção de gases desenvolvida por Theodorou et al. (1994) caracteriza-se pela leitura manual do volume de gases produzidos através de uma seringa plástica graduada. $\mathrm{O}$ uso da seringa restringe $\mathrm{o}$ número de amostras analisadas por experimento, diminui o número de leituras e consequentemente compromete descrição da curva de fermentação principalmente durante o período inicial de fermentação (lag-phase) e muitas vezes compromete a acurácia das leituras devido a erros cometidos pelo operador.

A técnica in vitro semi-automática de produção de gases (Mauricio et al., 1999) apresenta comprovado potencial em descrever a cinética da fermentação no rúmen, fornecer a taxa e a extensão da degradação das forrageiras (Getachew et al., 1998), bem como medir produtos da fermentação de partes solúveis e insolúveis de substratos (Pell \& Schofield, 1993). Essa técnica permite avaliar grande número de substratos por experimento, apresentando alta acurácia nas medições, simplicidade no manuseio de equipamentos e baixo custo na implantação e por amostra analisada.

O objetivo deste trabalho foi avaliar o potencial da técnica in vitro semi-automática de produção de gases para avaliação de silagens de sorgo pela comparação com dados obtidos em experimento in vivo.

\section{Material e Métodos}

Utilizaram-se quatro híbridos deSorghum bicolor (L.) Moench, sendo dois forrageiros, BR601 e AG2002, e dois de duplo propósito, BR700 e BR701. Os cultivares foram produzidos nas dependências da EMBRAPA - Milho e Sorgo, Sete Lagoas-MG. Este material foi ensilado em tambores com capacidade para $200 \mathrm{~L}$, abertos após 56 dias de fermentação para ensaio de digestibilidade aparente (in vivo), conforme descrito por Martins (2000). Nas amostras présecas, foram determinados o teor de matéria seca (MS), de acordo com método da AOAC (1990); os níveis de proteína bruta (PB), pelo método de Kjeldahl (AOAC, 1990); e os componentes da parede celular (FDN, FDA), pelo método seqüencial (Van Soest et al., 1991), utilizando-se $2 \mathrm{~mL}$ por amostra de uma solução de amilase $1 \%$, na determinação do FDN. Determinou-se o $\mathrm{pH}$ no suco da silagem, utilizando-se potenciômetro "Beckman Expandomatic SS-2" com escala expandida. $\mathrm{O}$ teor de $\mathrm{N}-\mathrm{NH}_{3} / \mathrm{NT}$ foi dosado imediatamente após a extração do suco, através da destilação com óxido de magnésio e cloreto de cálcio (AOAC, 1990). Parte representativa desse material foi amostrada para o ensaio in vitro, por intermédio da técnica de produção de gases e digestibilidade in vitro da matéria seca (DIVMS), segundo Tilley \& Terry (1963), sofrendo pré-secagem em estufa de ventilação forçada a $65^{\circ} \mathrm{C}$ e moagem em moinho com peneira de um milímetro.

Um grama de amostra foi adicionado aos frascos de fermentação $(160 \mathrm{~mL})$ previamente injetados com $\mathrm{CO}_{2}$. Foram utilizados três frascos por tratamento. Frascos contendo somente líquido ruminal e meio de cultura (tampão) foram usados como controle. Para cada frasco, foram adicionados manualmente, utilizando uma proveta, $90 \mathrm{~mL}$ de meio de cultura conforme, Theodorou et al. (1994). Os frascos foram vedados com rolhas de borracha $(14 \mathrm{~mm})$ e seladas com anilhas de alumínio. Para evitar que qualquer tipo de fermentação ocorresse, os frascos foram mantidos a $4^{\circ} \mathrm{C}$ durante a noite. Cinco horas antes da inoculação, os frascos foram removidos da geladeira para estufa a $39^{\circ} \mathrm{C}$ até o momento da inoculação. A inoculação foi feita usando líquido ruminal obtido de um bovino da raça holandesa, fistulado, mantido em dieta à base de volumoso (silagem de sorgo) à vontade e $1 \mathrm{~kg}$ de concentrado por dia (20\% de PB). O líquido ruminal foi retirado e armazenado em garrafas térmicas previamente aquecidas. No laboratório, o líquido ruminal foi filtrado através de duas camadas de gazes de algodão sob injeção contínua de $\mathrm{CO}_{2}$ e mantido em banho maria a $39^{\circ} \mathrm{C}$. A inoculação foi realizada através da injeção de $10 \mathrm{~mL}$ do inóculo por frasco através de seringa plástica conectada a uma agulha $(0,6 \mathrm{~mm})$. A pressão originada pelos gases acumulados na parte superior dos frascos foi medida por

R. Bras. Zootec., v.32, n.4, p.1013-1020, 2003 
intermédio de um transdutor de pressão (tipo T443A, Bailey \& Mackey, Inglaterra) conectado em sua extremidade a uma agulha $(0,6 \mathrm{~mm})$. As leituras de pressão foram tomadas em maior freqüência durante o período inicial de fermentação e reduzidas posteriormente $(2,4,6,8,10,12,15,19,24,30,36,48,72 \mathrm{e}$ 96 h). A partir da inserção da agulha na tampa de borracha a pressão produzida no interior dos frascos foi lida no leitor digital. Os dados de pressão (P; psi $=$ pressão por polegada quadrada) foram utilizados para o cálculo do volume de gases produzidos através de equação matemática, $\mathrm{V}=-0,004$ (s.e. 0,06) +4,43 $\mathrm{P}$ (s.e. 0,043) + 0,051 P2 (s.e. 0,007), desenvolvida por Mauricio et al. (2003).

Os resíduos de fermentação foram obtidos por filtragem em cadinhos de porosidade 1 (Pirex Vidrotec), os quais foram secados por 48 horas a $100^{\circ} \mathrm{C}$ e pesados para que fossem calculados os valores de degradabilidade da matéria seca (DMS). Esse procedimento foi realizado após 6, 12, 24, 48 e 96 horas de fermentação. Foram determinadas as correlações entre os resultados in vitro e in vivo (SAS, 1990).

Foi utilizado o modelo $\mathrm{Y}=\mathrm{A}\{1-\exp [-\mathrm{b}(\mathrm{t}-\mathrm{L})-$ c x $(\sqrt{ } t-\sqrt{ } L)]\}$ (1) de France et al. (1993), sendo $\mathrm{Y}=$ produção cumulativa de gases $(\mathrm{mL})$, $\mathrm{A}=$ assíndota ou potencial máximo de produção de gases, $\mathrm{L}=$ tempo de colonização (lag time), $\mathrm{b}\left(\mathrm{h}^{-1}\right)$ e c $\left(\mathrm{h}^{-0,5}\right)=$ taxas fracionais constantes. A taxa fracional $\left(\mathrm{h}^{-1}\right)$ combinada a produção de gases $(\mu)$ foi calculada sendo $\mu=b+c / 2 \sqrt{t}$ (2) onde, $\mu=$ taxa de produção de gases (h-1), b e c = parâmetros semelhantes ao da equação (1), $t$ = tempo de incubação em horas. As degradabilidades efetivas das matérias secas (DEMS) empregando as taxas de passagem de 2,5 e $8 \%$ /h para baixo, médio e alto consumo, respectivamente, conforme recomendações do Report.... (1984), foram calculadas pela equação (1) proposta por France et al. (1993), utilizando o software MLP (Ross, 1980), sendo
DEMS $=\mathrm{S}_{0} \mathrm{e}^{-\mathrm{kT}}\left(1-\mathrm{k}_{\mathrm{I}}\right) /\left(\mathrm{S}_{0}+\mathrm{U} 0\right)$, em que $\mathrm{k}=$ taxa de passagem, $S_{0}$ e $U_{0}=$ frações inicialmente fermentáveis e frações não fermentáveis.

\section{Resultados e Discussão}

Os resultados da análise bromatológica das silagens dos quatro híbridos de sorgo podem ser observados na Tabela 1 .

A silagem do Sorgo BR700 foi a que apresentou o maior teor de MS entre as silagens testadas (35,76\%), seguida pelo BR701 (35,02\%), BR601 $(29,74 \%)$ e AG2002 $(24,86)$. Estes resultados estão condizentes com o observado na literatura (Borges, 1998; Corrêa, 1996; Sousa et al., 2001; Molina et al., 2002) e estão próximos à margem de variação de 25 a $35 \%$, indicados por Nogueira (1995) como teores ótimos para a obtenção de uma fermentação predominantemente homolática.

Como pode ser observado na Tabela 1, os valores de PB variaram de 7,01 para o BR601 a 7,95\% para o BR700. Todas as silagens do presente experimento tiveram teores de proteína superiores ao mínimo de $7 \%$ recomendado por Silva et al. (1999) para efetiva fermentação microbiana no rúmen (Tabela 1).

Os valores de FDN e FDA variaram de 56,55 a $64,33 \%$ e de 41,34 a $53,75 \%$, respectivamente. Estes valores foram semelhantes a outros encontrados em literatura (Sousa et al., 2001; Molina et al., 2002). O híbrido BR601 foi o que obteve maiores teores de hemicelulose, podendo ser indicativo de superioridade durante a fermentação in vitro. Entretanto, a hemicelulose é um polissacarídeo complexo e sua digestibilidade é dependente da composição dos vários açucares formadores e do tipo de ligação glicosídica existente (Van Soest, 1994).

Os valores de $\mathrm{pH}$ das silagens dos híbridos avaliados variaram entre 3,9 e 4,2, resultados que podem ser considerados satisfatórios segundo critérios cita-

Tabela 1- Valores de MS, PB, FDN, FDA, hemicelulose, $\mathrm{pH}$, e $\mathrm{NH}_{3} / \mathrm{NT}$ das silagens de quatro híbridos de sorgo Table 1 - Values for dry matter (DM), crude protein (PB), neutral detergent fibre (NDF), acid detergent fibre (ADF), hemicellulose, $\mathrm{pH}$ and amoniacal nitrogen $\left(\mathrm{NH}_{3} / \mathrm{NT}\right)$ for silages of four sorghum hybrids

\begin{tabular}{|c|c|c|c|c|c|c|c|}
\hline $\begin{array}{l}\text { Híbridos } \\
\text { Hybrids }\end{array}$ & $\begin{array}{l}\mathrm{MS} \\
D M\end{array}$ & $\begin{array}{l}\text { PB } \\
P B\end{array}$ & $\begin{array}{l}\mathrm{FDN} \\
N D F\end{array}$ & $\begin{array}{l}\text { FDA } \\
A D F\end{array}$ & $\begin{array}{l}\text { Hemicelulose } \\
\text { Hemicellulose }\end{array}$ & $\begin{array}{l}\mathrm{pH} \\
\mathrm{pH}\end{array}$ & $\begin{array}{l}\mathrm{NH}_{3} / \mathrm{NT} \\
\mathrm{NH}_{3} / \mathrm{NT} \\
\end{array}$ \\
\hline Br700 & 35,8 & 8,0 & 56,6 & 53,8 & 2,8 & 4,0 & 3,4 \\
\hline Br701 & 35,0 & 7,5 & 64,3 & 47,6 & 16,7 & 3,9 & 3,8 \\
\hline Br601 & 29,7 & 7,0 & 61,1 & 41,3 & 19,7 & 3,9 & 4,7 \\
\hline AG2002 & 24,9 & 7,1 & 59,8 & 46,6 & 13,2 & 4,2 & 2,7 \\
\hline
\end{tabular}

R. Bras. Zootec., v.32, n.4, p.1013-1020, 2003 
dos por Borges et al. (1998). Quanto aos teores de $\mathrm{NH}_{3}$ /NT os quatro híbridos apresentaram valores abaixo de $10 \%$, indicando que o processo fermentativo não resultou na quebra excessiva de proteína, com a conseqüente geração de amônia (Martins, 2000).

Na Figura 1, podem ser observados os gráficos das equações de regressão entre a produção cumulativa de gases e DMS para cada um dos híbridos avaliados e para os quatro híbridos agrupados.

Como podem ser observadas, as altas correlações (BR700 $\mathrm{r}^{2}=0,99$; BR701 $\mathrm{r}^{2}=0,99$; BR601 $\mathrm{r}^{2}=0,99$; AG2002 $\mathrm{r}^{2}=0,98$ ) entre os resultados da DMS nos tempos $6,12,24,48$ e $96 \mathrm{~h}$ e as respectivas produções de gases $(\mathrm{mL})$ refletem a relação entre o volume de gases produzido e o processo de degradação da matéria seca. Estas elevadas correlações também já haviam sido descritas por Mould et al. (1999) ao trabalhar com silagens de milho.

Quando todos os dados de produção de gases dos quatro híbridos foram correlacionados com as respectivas DMS (Figura 1), a equação obtida foi semelhante as anteriores $\left(\mathrm{r}^{2}=0,99\right)$. Estes resultados demonstram a possibilidade de estimar a curva de degradação da MS através dos valores da produção cumulativa de gases para grupos específicos de alimentos (ex. silagem de sorgo).

Segundo Beuvink \& Spoelstra (1992), a relação propionato/acetato pode interferir no volume de gases produzidos, onde a maior produção de acetato favorece a produção de gases. Existe uma interação entre os ácidos graxos voláteis e o meio de cultura (tampão), em que o decréscimo no $\mathrm{pH}$ favorece a produção de propionato e, conseqüentemente, redução na produção de gases. Todavia, neste experimento, a possível presença dos produtos de degradação (AGV) dos materiais estudados não interferiu na relação entre degradação e produção de gases.

Na Figura 2, podem ser observadas as curvas de produção cumulativa de gases das silagens dos quatro híbridos de sorgo avaliados, que refletem a degradação do material. Nota-se, graficamente, a superioridade do híbrido BR 601 seguido pelos híbridos BR700 e BR701, que apresentaram curvas similares, e AG2002.

Na Figura 3, observa-se o gráfico das taxas de produção de gases das silagens dos quatro híbridos de sorgo avaliados nos diferentes tempos de fermentação. As maiores taxas de produção de gases foram obtidas, para todos os híbridos, aproximadamente após oito horas de fermentação, fato provavelmente ligado à fermentação dos carboidratos solúveis. Observa-se entre os períodos de fermentação de, aproximadamente, 16 a 24 horas elevação na taxa de produção de gases, que provavelmente está relacionada à fermentação dos carboidratos fibrosos. Verifica-se também a superioridade da taxa de fermentação do BR601, quando comparado aos demais híbridos.

$\mathrm{Na}$ Tabela 2, estão descritos os parâmetros de produção de gases estimados pelo modelo de France et al. (1993), produção total de gás (PTG), digestibilidade in vitro da matéria seca após 48 e 96 horas de fermentação (DMS48 e DMS96, respectivamente), digestibilidade in vitro da matéria seca (DIVMS) segundo Tilley \& Terry (1963) e digestibilidade in vivo da matéria seca (DMS in vivo). Comparando-se os valores de DMS 48, DMS 96, DMS in vivo e DIVMS, verifica-se que o BR 601 foi o híbrido que apresentou os maiores valores, seguido dos híbridos BR700, BR701 e AG2002, os quais apresentaram valores próximos entre si. Associando-se os valores de DMS 48, DMS 96, DMS in vivo e DIVMS com os resultados da análise bromatológica das silagens (Tabela 1), observa-se que o BR601 foi o híbrido que apresentou os menores valores de FDA. Em parte este fato está relacionado à característica de seu colmo, uma vez que o conteúdo de FDA geralmente está correlacionado negativamente com a digestibilidade das forragens.

Comparando-se os dados obtidos no experimento in vitro (Produção Total de Gás = PTG) com os obtidos in vivo (DMS in vivo) foi encontrada a seguinte equação: digestibilidade in vivo $(\mathrm{g} / \mathrm{kg})=$ 0,46PTG $(\mathrm{mL})+361,08\left(\mathrm{r}^{2}=0,97\right)$, mostrando a proximidade dos valores in vitro em relação aos in vivo. Vale ressaltar que os dados da DMS obtidos a $48 \mathrm{~h}$ foram numericamente mais próximos aos valores in vivo que os valores obtidos a $96 \mathrm{~h}$. Provavelmente este resultado está associado ao período de permanência do alimento no rúmen (48 h aproximadamente).

Quanto aos parâmetros de France et al. (1993), os quais descrevem numericamente a cinética de fermentação ruminal (Tabela 2), nota-se que o potencial máximo de produção de gases (A), parâmetro este que representa a máxima produção de gases quando a curva atinge o seu platô, foi superior para o BR601. Para os parâmetros "lag phase" e taxa de fermentação $(\mu)$, nota-se que apesar da proximidade dos valores das "lag phase", o híbrido BR601 apresentou a maior taxa de fermentação $(\mu)$, fato provavel-

R. Bras. Zootec., v.32, n.4, p.1013-1020, 2003 
Gás (Gas) x DMS (BR700)

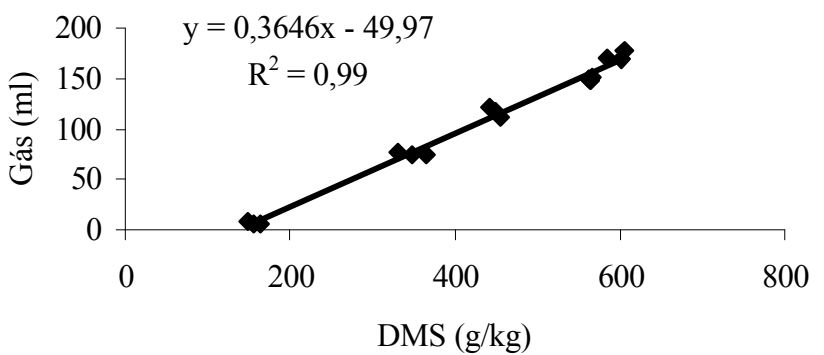

Gás (Gas ) x DMS (BR601)

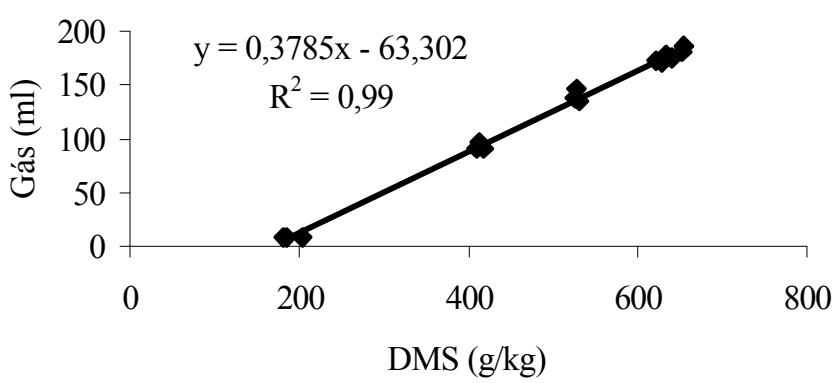

Gás (Gas ) x DMS (BR701)

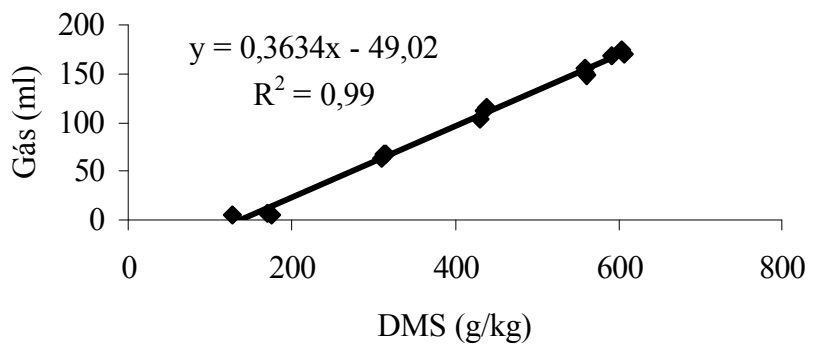

Gás (Gas) x DMS (AG2002)

$\mathrm{D}$

Gás (Gas ) x DMS (4 Genótipos; 4 Genotyps ) E

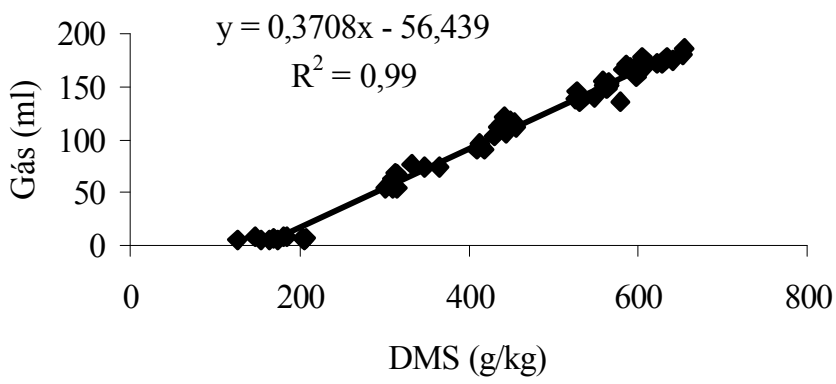

Figura 1 - Equações de regressão entre produção de gases e DMS para cada híbrido $(A=B R 700, B=B R 701, C=B R 601$, $\mathrm{D}=\mathrm{AG} 2002)$ e para os quatro híbridos agrupados ( $\mathrm{E}=\mathrm{BR700}$, BR701, BR601 e AG2002).

Figure 1 - Regression equations related to gas and $D M D$ for each genotype $(A=B R 700, B=B R 701, C=B R 601, D=A G 2002)$ and also for all the genotypes together ( $E=B R 700, B R 701, B R 601$ and AG2002).

mente associado aos maiores teores de substratos prontamente fermentáveis na silagem desse híbrido, evidenciando, dessa forma, a superioridade no valor da DMS 48 e 96 (628 e $649 \mathrm{~g} / \mathrm{kg}$, respectivamente).

Os trabalhos do Report... (1984) recomendam que as taxas de passagem de 2,5 e $8 \% / \mathrm{h}$ sejam empregadas para o cálculo da degradabilidade efetiva em condições em que as taxas de passagem não foram ou não puderam ser medidas. Adotam-se taxas de $2 \% / \mathrm{h}$ para bovinos e ovinos em mantença; $5 \% / \mathrm{h}$ são próprios para vacas leiteiras de pequena produ- ção $(<15 \mathrm{~kg}$ de leite/dia) bovinos de corte e ovinos em crescimento quando usada dieta mista; enquanto a taxa de $8 \% / \mathrm{h}$ é recomendada para vacas produzindo acima de $15 \mathrm{~kg}$ de leite/dia e alimentadas com dietas mistas.

$\mathrm{Na}$ Tabela 3, são apresentados os valores de degradação efetiva da MS (DEMS) estimados para as taxas de passagens de 2,5 e $8 \% /$ h. Como pode ser observado, os valores de degradabilidade efetiva confirmama superioridade da silagem do híbrido BR601 para todas as taxas de passagem. 
Tabela 2 - Parâmetros de produção de gases estimados pelo modelo de France et al. (1993) ${ }^{1}$; produção total de gases (PTG), digestibilidade in vitro da matéria seca a $48 \mathrm{~h}$ (DMS 48) e a $96 \mathrm{~h}$ (DMS 96), digestibilidade in vitro (DIVMS) e in vivo (DMS in vivo) da matéria seca das silagens de quatro híbridos de sorgo

Table 2 - Gas production parameters estimated from France et. (1993) ${ }^{1}$ model; total gas production (PTG), in vitro dry matter digestibility at 48 or $96 \mathrm{~h}$ (DMS 48, DMS 96), in vitro (DIVMS) and in vivo (DMS in vivo) dry matter digestibility of silages from four hybrids of sorghum

\begin{tabular}{|c|c|c|c|c|c|c|c|c|c|c|}
\hline Genótipos & $\mathrm{A}^{2}$ & & $\mathrm{~L}(\mathrm{~h})^{3}$ & & $\mathrm{~m}(/ \mathrm{h})^{4}$ & PTG & DMS 48 & DMS 96 & DIVMS $^{5}$ & DMS in vivo ${ }^{6}$ \\
\hline Genotypes & & s.e & & s.e & & $(\mathrm{ml})$ & $\mathrm{g} / \mathrm{kg}$ & $\mathrm{g} / \mathrm{kg}$ & $\mathrm{g} / \mathrm{kg}$ & $\mathrm{g} / \mathrm{kg}$ \\
\hline $\mathrm{Br} 700$ & 179 & 4,4 & 2,3 & 0,4 & 0,051 & 179 & 565 & 598 & 582 & $522_{\mathrm{B}}$ \\
\hline BR701 & 179 & 3,4 & 2,2 & 0,4 & 0,044 & 178 & 560 & 601 & 580 & $505_{B}^{\mathrm{D}}$ \\
\hline BR601 & 194 & 5,2 & 2,4 & 0,5 & 0,056 & 198 & 628 & 649 & 631 & $618_{\mathrm{A}}^{\mathrm{B}}$ \\
\hline AG2002 & 166 & 2,7 & 2,1 & 0,4 & 0,045 & 167 & 564 & 593 & 564 & $498_{\mathrm{B}}^{\mathrm{A}}$ \\
\hline
\end{tabular}

1 Modelo de France et al. (1993): $G=A-B Q^{t} Z{ }^{t}$

2 Potencial máximo de produção de gás.

3 "Lag phase".

4 Taxa de produção de gás.

5 Digestibilidade in vitro da matéria seca (Tilley \& Terry, 1963).

6 Letras maiúsculas distintas em uma mesma coluna representam diferença estatística $(p<0,05$; teste SNK). Dados obtidos de Martins (2000).

1 France et al. (1993) model.

2 Potential of gas production.

4 Fractional rate of fermentation.

5 In vitro dry matter digestibility (Tilley \& Terry, 1963).

${ }^{6}$ Different subscripts within the same column indicate significant differences $(p<0,05)$. Data obtained from Martins $(2000)$.

$\mathrm{Na}$ literatura, os valores relatados de degradabilidade efetiva para silagem de sorgo são obtidos por intermédio da técnica in situ, sendo geralmente superiores aos encontrados nesse experimento pela técnica de produção de gases para as mesmas taxas de passagem $(2,5$ e $8 \% / h)$. Utilizando a técnica in situ, Rabelo (1997) encontrou para híbridos de sorgo com altos teores de tanino, valores de 51,03, 40,17 e $35,42 \%$, próximos aos encontrados por Molina et al. (2002), utilizando-se a mesma técnica, o qual

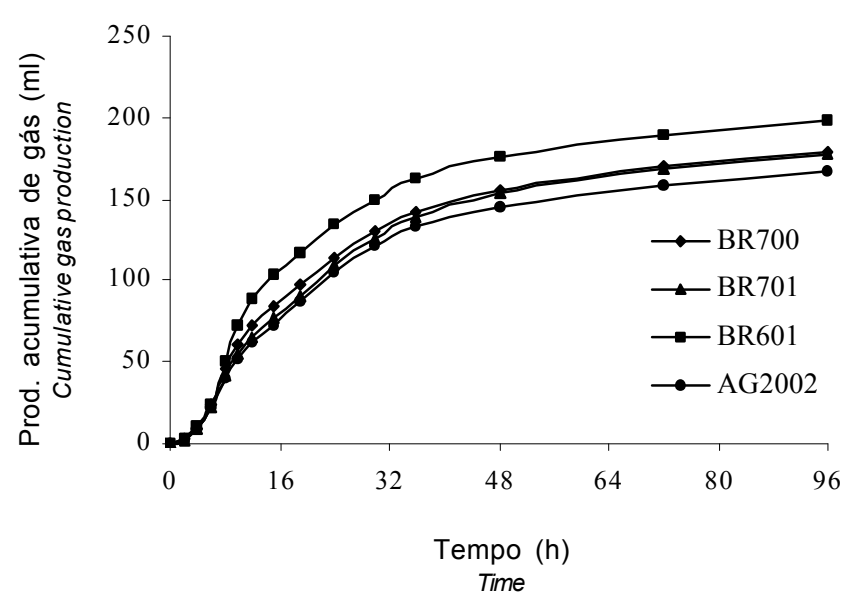

Figura 2 - Curvas de produção cumulativa de gases das silagens de quatro híbridos de sorgo.

Figure 2 - Cumulative gas production profiles from silages of four genotypes of sorghum. relatou 52,80,39,12 e 32,51\% para o híbrido BR700, que também foi utilizado neste experimento. Estes resultados sugerem que sejam realizados novos experimentos comparando a degradabilidade efetiva obtida por intermédio das técnicas in situ e a produção de gases.

McDonald et al. (1995) obtiveram alta correlação entre digestibilidade e consumo de matéria seca. Entretanto, o NRC (2001) sugere redução no valor energético dos alimentos em até $8 \%$, à medida que o consumo aumenta.

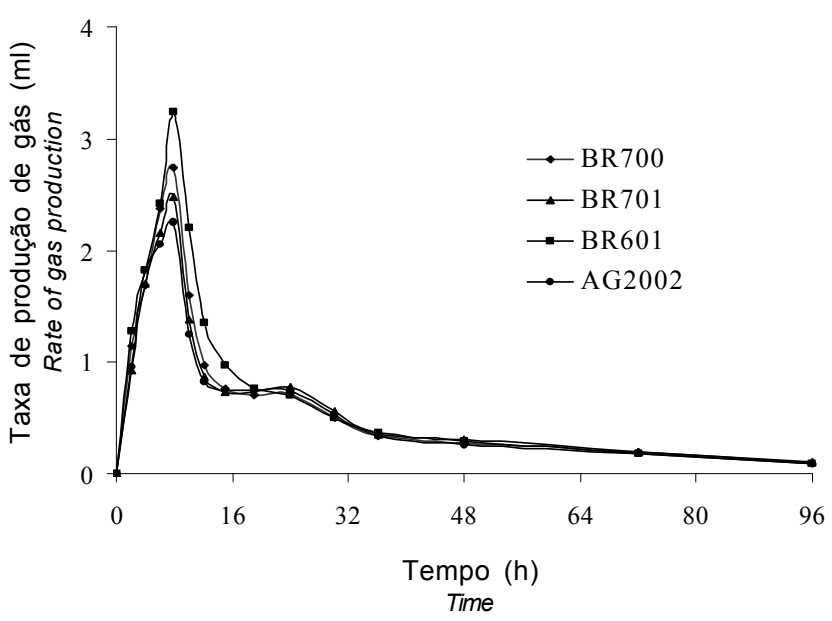

Figura 3 - Taxa de produção de gases das silagens de quatro híbridos de sorgo.

Figure 3 - Rate of gas poduction from silages of four genotypes of sorghum.

R. Bras. Zootec., v.32, n.4, p.1013-1020, 2003 
Tabela 3- Valores da degradação efetiva da MS (DEMS) obtidos para as taxas de passagens 2, 5 e 8 $\% / \mathrm{h}$ obtidos através da técnica semi-automática de produção de gases

Table 3 - Values for the extent of degradation in the rumen using the rates of passages 2, 5 e $8 \% / h$ obtained though semi-automated gas production technique

\begin{tabular}{|c|c|c|c|}
\hline \multirow[t]{2}{*}{$\begin{array}{l}\text { Híbridos } \\
\text { Hybryds }\end{array}$} & \multicolumn{3}{|c|}{$\begin{array}{c}\text { Taxas de passagem }(\% \mathrm{H}) \\
\text { Passage rates }\end{array}$} \\
\hline & 2 & 5 & 8 \\
\hline BR700 & 425 & 300 & 234 \\
\hline BR701 & 415 & 287 & 222 \\
\hline BR601 & 478 & 343 & 267 \\
\hline AG2002 & 418 & 293 & 229 \\
\hline
\end{tabular}

Já Blümmel e Ørskov (1993), trabalhando com resultados obtidos por intermédio da técnica de produção de gases de Menke et al. (1979) e sacos de náilon sugeriram que a taxa de fermentação $(\mu)$ e a taxa de degradação da matéria seca (c), respectivamente, proporcionaram coeficientes de correlação com consumo superiores aos obtidos em experimentos de digestibilidade aparente. Dessa forma, os parâmetros de France et al. (1993), principalmente m, obtidos neste experimento indicam a possibilidade de que a silagem do híbrido BR601 proporcione maior consumo, provavelmente devido ao seu maior potencial nutritivo para os ruminantes, fato comprovado pelo ensaio de digestibilidade in vivo.

Os resultados deste experimento demonstraram o potencial da técnica in vitro semi-automática de produção de gases para avaliação de silagens de sorgo. Esta técnica, por apresentar baixo custo, alta repetibilidade, capacidade de avaliar elevada quantidade de substratos (híbridos) e ainda possibilitar estimativas acuradas de experimentos in vivo, pode tornar-se importante ferramenta de balizamento do melhoramento genético de híbridos de sorgo para produção de silagens.

\section{Conclusões}

A técnica in vitro semi-automática de produção de gases possibilitou a estimativa dos valores de digestibilidade aparente in vivo e forneceu informações adicionais sobre a cinética de fermentação ruminal das silagens avaliadas.

Entre os híbridos avaliados, o BR601 apresentou-se como o mais promissor.

\section{Literatura Citada}

ASSOCIATION OF OFFICIAL ANALYTICAL CHEMISTS AOAC. AGRICULTURAL CHEMISTS. Official methods of analysis. 16.ed. Washington, D.C.: 1990. 1094p.

BORGES, A.C.C.; RODRIGUEZ, N.M.; GONÇALVES, L.C. et al. Silagens de sorgo de porte alto com diferentes teores de tanino e umidade no colmo. I - Matéria seca, proteína bruta e balanço de nitrogênio. Arquivos Brasileiros de Medicina Veterinária e Zootecnia, v.49, p.709-714, 1998.

BEUVINK, J.M.W.; SPOELSTRA, S.F. Interactions between substrate, fermentation end-products, buffering systems and gas production upon fermentation of different carbohydrates by mixed rumen microorganisms in vitro. Applied Microbiology and Biotechnology, v.37, p.505-509,1992.

BLÜMMEL, M.; ØRSKOV, E.R. Comparison of "in vitro" gas production and naylon degradability of roughage in predicting feed intake in cattle. Animal Feed Science and Technology, v.40, p.109-119, 1993

CASELA, C.R.; BORGONOVI, R.A.; SCHAFFERT, R.E. et al. Cultivares de sorgo. Informe Agropecuário, v.12, n.144, p.40-43, 1986.

CORRÊA, C.E.S.; RODRIGUES, J.A.S.; GONÇALVES, L.C. et al. Determinação da produção de matéria seca e das proporções de colmo, folha e panícula de treze híbridos de sorgo. In: REUNIÃO ANUAL DA SOCIEDADE BRASILEIRA DE ZOOTECNIA, 33., 1996, Fortaleza. Anais... Fortaleza: Sociedade Brasileira de Zootecnia, 1996.

FRANCE, J.; DHANOA, M.S.; THEODOROU, M.K. A model to interpret gas accumulation profiles with "in vitro" degradation of ruminants feeds. Journal of Theoretical Biology, v.163, p.99-111, 1993.

MARTINS, R.M. Consumo e digestibilidade aparente das silagens de quatro genótipos de sorgo (Sorghum bicolor (L.) Moench). Belo Horizonte: Universidade Federal de Minas Gerais, 2000. 70p. Dissertação (Mestrado em Zootecnia) - Universidade Federal de Minas Gerais, 2000.

MAURICIO, R.M.; MOULD, F.L.; DHANOA, M.S. et al. A semi-automated in vitro gas production technique for ruminants feedstuff evaluation. Animal Feed Science Technology, v.79, p.321-330,1999.

MAURICIO, R.M.; PEREIRA, L.G.R.; GONÇALVES, L.C. et al. Relação entre pressão e volume para implantação da técnica in vitro semi-automática de produção de gases na avaliação de forrageiras tropicais. Arquivos Brasileiros de Medicina Veterinária e Zootecnia, v.55, p.216-219, 2003.

McDONALD, P.; EDWARDS, R.A.; GREENHALGH, J.F.D. et al. Animal nutrition. 5.ed. Singapore: Longman Scientific Technical, 1995. 607p.

MENKE, K.H.; RAAB, L.; SALEWSKI, A. et al. The estimation of the digestibility and metabolizable energy content of ruminant feeding stuffs from the gas production when they are incubated with rumen liquor in vitro. Journal of Agricultural Science, v.93, p.217-222, 1979.

MOLINA, L.R.; RODRIGUEZ, N.M.; GONÇALVES, L.C. et al. Degradabilidade in situ da matéria seca e da proteína bruta das silagens de seis genótipos de sorgo [Sorghum bicolor (L.) Moench], com e sem tanino no grão, ensilados no estádio de grão farináceo. Brazilian Journal of Veterinary Research and Animal Science, v.39, n.1/6, p.233-237, 2002.

MOULD, F.L.; SMITH, T.; OWEN, E. et al. The relationship between DOMD and gas release estimated "in vitro" using the reading pressure technique system for four maize silages 
of different maturity. Proceedings of the British Society of Animal Science, 1999. p. 150.

NOGUEIRA, F.A.S. Qualidade de silagens de híbridos de sorgo de porte baixo com e sem tanino e de colmo seco e suculento, e seus padrões de fermentação, em condições de laboratório. Belo Horizonte: Universidade Federal de Minas Gerais, 1995. 78p. Dissertação (Mestrado em Zootecnia) - Universidade Federal de Minas Gerais, 1995.

NATIONAL RESEARCH COUNCIL - NRC. Nutrient requirements for dairy cattle. 7.ed. Washington, D.C.: National Academy Press, 2001. 362p.

ØRSKOV, E.R.; HOVELL, F.D.B.; MOULD, F. The use of the nylon bag technique for evaluation of feedstuffs. Tropical Animal Production, v.5, p.195-213, 1980.

RABELO, E. Degradabilidade "in situ"de silagens de híbridos de sorgo [Sorghum bicolor (L.) Moench] de porte médio com diferentes teores de taninos e suculência no colmo. Belo Horizonte: Universidade Federal de Minas Gerais, 1997. 98p. Dissertação (Mestrado em Zootecnia) Universidade Federal de Minas Gerais, 1997.

REPORT of the protein group of the Agrucultural Research Council Working party, on the nutrient of ruminants. London. Commonwealth Agricultural Bureaux, 1984. 45p.

ROSS, G.J.S. Maximun Likelihood Program (A Manual). Tothmsted Experimental Station, Hampendon. 1987.

SILVA, F.F.; GONÇALVES, L.C.; RODRIGUES, J.A.S. et al. Qualidade das silagens de híbridos de sorgo (Sorghum bicolor (L.) Moench). de portes baixo, médio e alto com diferentes proporções de colmo mais folhas/panícula. I. Avaliação do processo fermentativo. Revista Brasileira de Zootecnia, v.28, n.1, p.21-29, 1999.

SOUSA, B.M.; SATURNINO, H.M.; CAMPOS, W.E. et al. Parâmetros de degradação in situ de silagens de três genótipos de sorgo com diferentes concentrações de tanino no grão. 1. Matéria seca e proteína bruta. In: REUNIÃO ANUAL DA SOCIEDADE BRASILEIRA DE ZOOTECNIA, 38., 2001, Piracicaba. Anais... Piracicaba: Sociedade Brasileira de Zootecnia, 2001. v.1, p.1167-1168.
SAS INSTITUTE . User's Guide: Statistics. 4.ed. Cary: 1990. $956 \mathrm{p}$.

THEODOROU, M.K.; WILLIAMS, B.A.; DHANOA, M.S. et al. A simple gas production method using a pressure transducer to determine the fermentation kinetics of ruminant feeds. Animal Feed Science and Technology, v.48, p.185-197, 1994.

TILLEY, J.M.A.; TERRY, R.A. A two-stage technique for the in vitro digestion of forage crops. Journal British Grassland Society, v.18, p.104-111, 1963.

Van SOEST, P.J.; ROBERTSON, J.B.; LEWIS, B.A. Methods for dietary fiber, neutral detergent, and nonstarch polysaccharides in relation to animal nutrition. Journal of Dairy Science, v.74, n.10, 3583-3597, 1991.

Van SOEST, P.J. Nutritional ecology of the ruminant. 3.ed. Ithaca: Cornell University Press, 1994. 476p.

ZAGO, C.P. Cultura de sorgo para produção de silagem de alto valor nutritivo. In: SIMPÓSIO SOBRE NUTRIÇÃO DE BOVINOS, 4., 1991, Piracicaba. Anais... Piracicaba: Fundação de Estudos Agrários "Luiz de Queiroz”, 1991. p.169-217.

ZAGO, C.P. Silagem de sorgo. In: SIMPÓSIO SOBRE NUTRIÇÃO DE BOVInOS, 7., 1999, Piracicaba. Anais... Piracicaba: Fundação de Estudos Agrários "Luiz de Queiroz”, 1999. p.47-68. 